\title{
Monitoring of the stress-strain state of open pits' adjacent rock mass
}

\author{
Andrey Panzhin ${ }^{1 *}$, Natalia Panzhina ${ }^{1}$ \\ ${ }^{1}$ Institute of Mining, Ural Branch of the Russian Academy of Sciences, 58 Mamina-Sibiryak Str., \\ Yekaterinburg city, Russia, 620075
}

\begin{abstract}
In the course of the work to identify the modern coordinates of the points of the state (SGN) and reference mine surveying (RMSN) networks of Orenburg Minerals JSC, the deformation characteristics of the earth's surface in different time periods were obtained and a geomechanical model of the rock mass was built both on the industrial site itself (quarry, dump), and beyond the areas, influenced by mining. Based on the data obtained, the parameters of deformation of the rock mass and the adjacent rock mass in three planes, as well as the relative deformations and the magnitudes of the tectonic stresses that caused them were identified; during the analysis of negative factors potentially dangerous areas of the adjacent rock mass were also identified.
\end{abstract}

\section{Introduction}

The territory on which the Kiembaevsk chrysotile-asbestos field is located is confined to the Main Ural fault zone and is represented by three main deposits: the Main site, the Northern site and the Third site. The most powerful tectonic zones at the Kiembaevsk field are separated by the three above-mentioned asbestos-bearing deposits. These tectonic zones are represented by a strip of varying thickness and mainly of a steep eastern dip, the strike of the zones being submeridional and sublatitudinal. In general, the tectonic structure of the area of the field and the enclosing rock mass allows predicting a high level of modern geodynamic mobility outside the area of significant anthropogenic impact, while the displacements and deformations of the background can be comparable to anthropogenic displacements and deformations.

The main, and often the only, source of data on the initial and anthropogenically altered stress-strain state of the rock mass is field measurements of the displacement process parameters [1]. In this case, rock displacement, a phenomenon that accompanies the extraction of a mineral, is understood as the whole complex of deformation processes occurring in the rock mass, in this case in the adjacent rock mass, during the formation of the initial stress-strain state outside the area of influence of mining and its transformation within it.

The main factors determining the formation of the stress-strain state of the rock mass are:

- hierarchically block structure;

\footnotetext{
*Corresponding author: panzhin@igduran.ru
} 
- constant mobility;

- secondary structuring;

- concentration of modern geodynamic movements at the boundaries of secondary structural blocks.

\section{Methodology}

To identify the parameters of the initial and secondary changed stress-strain state of the rock mass at the Kiembaevsk field, the coordinates of the points of the state geodetic network (SGN) and the reference mine surveying network (RMSN) located in the study area were redefined. Instrumental observations in the field area were carried out by the Mining Institute of the UB RAS in 2006 and 2017 using the GNSS satellite geodetic system, while the repeated identification of the spatial coordinates of the GGS and OMS points made it possible to establish the magnitudes and directions of the displacement vectors, the amplitudes of deformations and the nature of their action, to identify the displacements and deformations in the vertical plane and integral (3D) ones, that occurred since the beginning of field development and over the past 10 years. Also, strain tensors and changes in tectonic stresses that caused these deformations were identified based on the data obtained on the displacement process [2, 3].

In order to identify the parameters of cyclic short-period geodynamic movements, measurements are carried out in the form of continuous monitoring by satellite geodesy complexes over the system of points for several hours. The measurements record the change in the spatial coordinates of the points and their components in the monitoring network [4]. Continuous observations are discretized at the required time intervals, for the duration of which the average values of the coordinate increments are found. The sampling interval can be from a few seconds to several tens of minutes. The limit of the fixed frequencies of cyclic movements depends on the duration of the interval. The smaller the interval, the more cycle rates are recorded by measurements [5].

Continuous monitoring by satellite geodesy complexes ensures obtaining of the increments of all three coordinates of the instrument's position point at a given moment in time. The accuracy of measuring displacements between points is 3-5 mm. The same accuracy as the one displayed in finding the trend movements is provided by the differential technology of satellite geodesy GPS, in which two or more simultaneously operating receivers installed at the ends of the measured segments allow identifying the value of the ionospheric correction, due to which the accuracy is achieved.

\section{Results}

The displacements of the rock mass, having linear dimensions of about 10x12 km, including the quarry and dumps of the Kiembaevsk chrysotile-asbestos field, range from 55 to $338 \mathrm{~mm}$ in plan view (2D) and integral (3D) being from 59 to $378 \mathrm{~mm}$ for the period from 1974 to 2017. The displacement vectors obtained as a result of instrumental measurements are shown in Fig. 1. There is a generally coordinated direction of the vectors in the direction of the mined open pit; however, clusters with predominant movements to the west (Slavensk, Poiskovy, Basis) and south (Podgorny, Ploskiy) in the northern part of the study area are distinguished. 


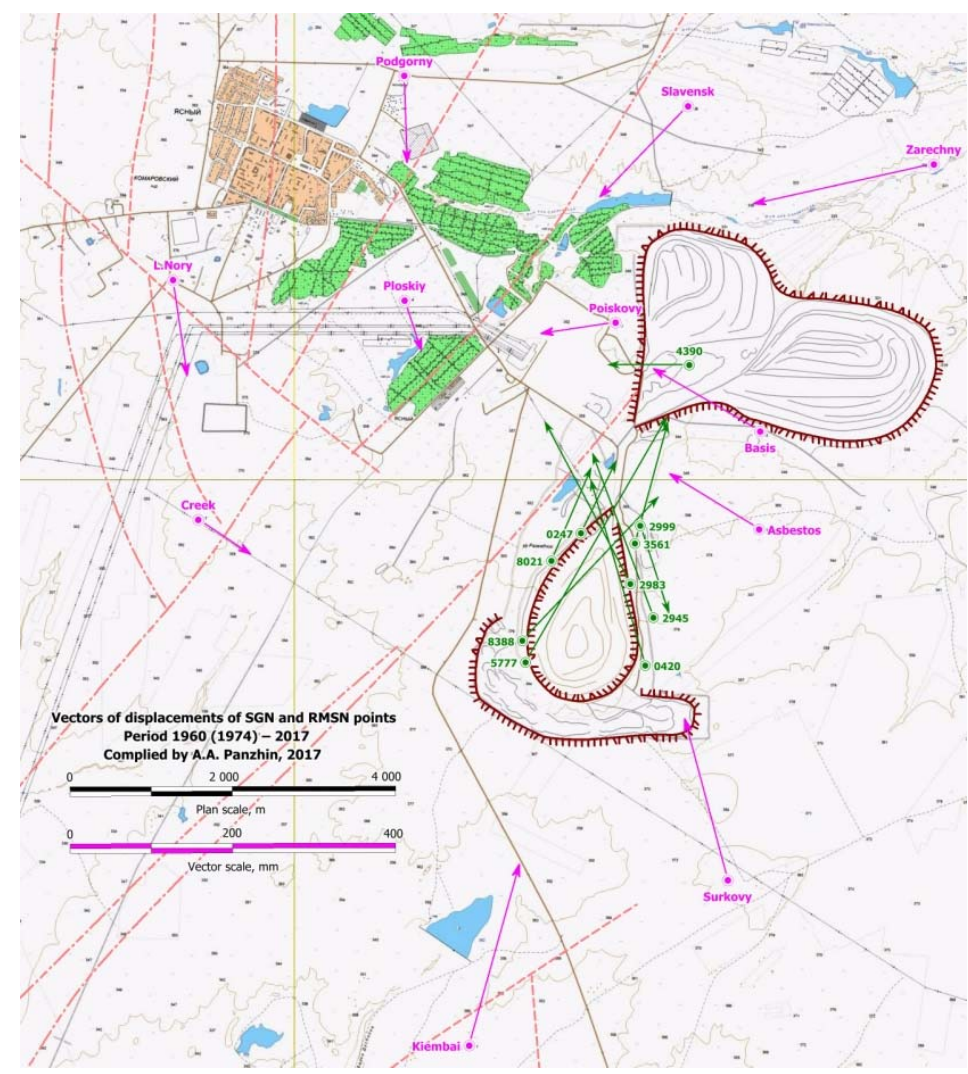

Fig. 1. Vectors of displacement of the rock mass for the period $1974-2017$

The strain tensors calculated on the basis of the values of the displacement vectors form a rather calm field in terms of magnitude and directions of action; compression deformations prevail in the area of the quarry space, without noticeable zones of their concentration, which may indicate a generally favorable forecast for the long-term stability of the pit walls.

The rock mass displacements recorded for the period 2006 - 2017 are much lower and range from 7 to $69 \mathrm{~mm}$ in plan (2D) and integral (3D) being from 14 to $124 \mathrm{~mm}$. The displacement vectors are shown in Fig. 2, and, as in the previous period, it shows a predominantly consistent direction of the vectors towards the mined open pit space.

To study the geomechanical state of the open pit rock mass, horizontal and vertical displacements of the SGN points were studied for the period 2006 - 2017. The displacements of the adjacent rock mass, having linear dimensions of $2 \times 2.7 \mathrm{~km}$, including the open pit, are from 18 to $88 \mathrm{~mm}$ in plan (2D) and integral (3D) being from 32 to $123 \mathrm{~mm}$ for the period from 2006 to 2017. The displacement vectors and movement vectors obtained as a result of instrumental measurements are shown in Fig. 2a. It is noted that with a predominantly consistent direction of vectors towards the mined pit space, some points that have displacements towards the rock mass, as well as points with displacement vectors differing from neighboring ones by 2-3 times, can be distinguished. The displacement vectors demonstrate a vortex distribution, and several of their sources are distinguished the areas of increased displacement values, located mainly in the western and southeastern sides of the open pit. 
a.

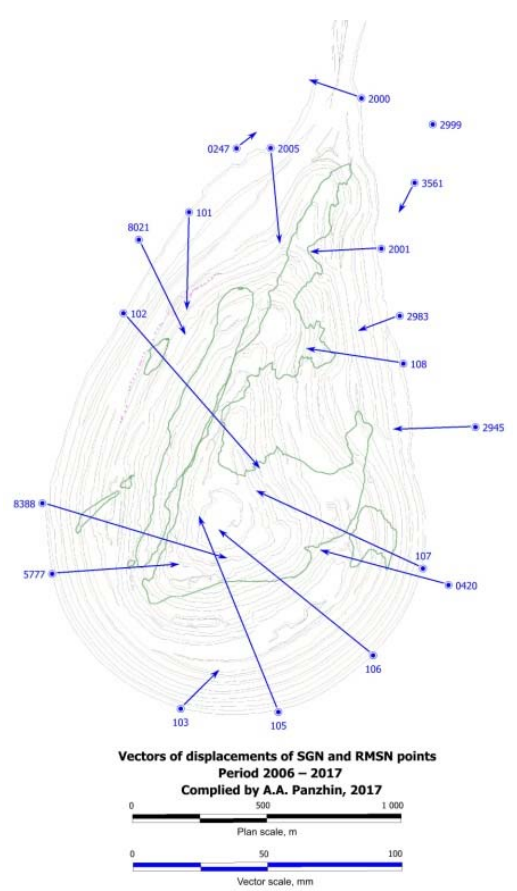

b.

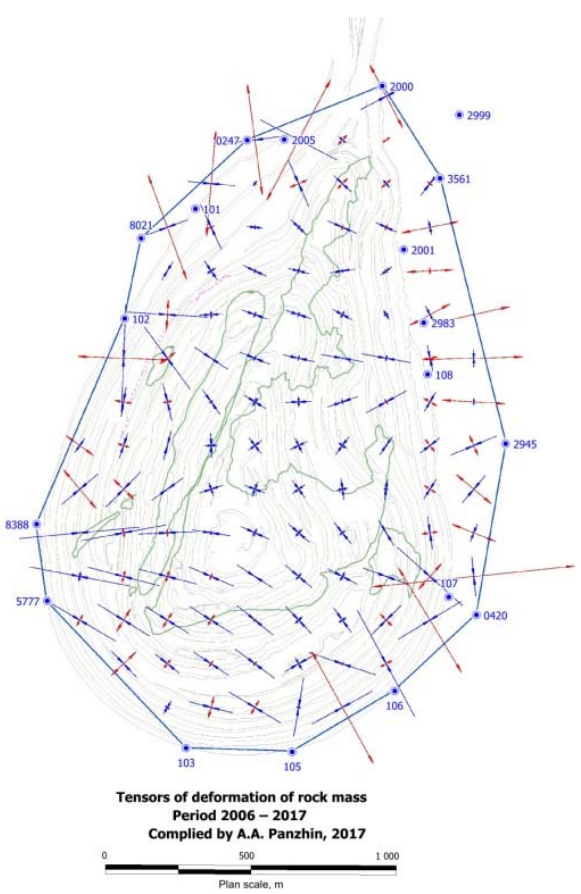

Fig. 2. Displacement vectors (a) and strain tensors (b) of the adjacent rock mass for the period 2006 (2007) - 2017

The strain tensors for this period (Fig.2b) form a field that is non-uniform in magnitude and directions of action; both deformations of compression (the southern part of the open pit) and extension (depression) prevail in the area of the open pit, which can negatively affect the stability of the pit walls and activation of the cracking process [6].

The parameters of cyclical geodynamic movements were investigated by the change in the vector components calculated between observation points located in the adjacent rock mass. Office processing of field observations was performed using the Motion Tracker module of the Trimble Total Control proprietary software in compliance with the authors' technique described in [5]. To control the processing of instrumental measurements, the Waypoint GrafNet/GrafNav package was also used, which supports powerful capabilities for calculating kinematic trajectories.

The accuracy of the methodology for studying the short-period geodynamics of the rock mass was verified by comparing the results of the office processing of measurements made in the adjacent rock mass by two series of instrumental observations, where the data on 4-5 benchmarks of the observation station was used. As a result of the experiment, 16 independent sets of vectors were obtained between the reference points, regarding which long-term continuous observations were made. The results of processing the initial data in various software packages are presented in the form of rose diagrams for the components "north-south" and "west-east” of short-period geodynamic movements (Fig. 3). 


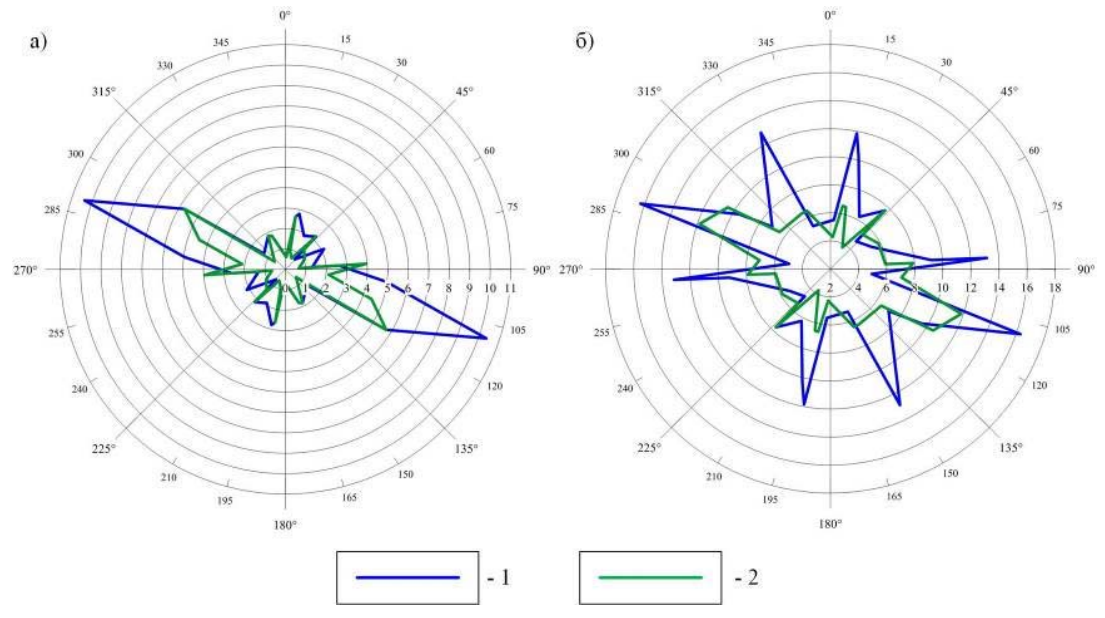

Fig. 3. Comparison of the results of office processing in the GrafNet/GrafNav software (a) and the Motion Tracker module of the Trimble Total Control software (b): 1 - north-south component; 2 - west-east component

Fig. 3 shows that according to the processing results using both software packages, the main direction of the action of horizontal short-period geodynamic movements along the components "north-south" and "west-east" is clearly recorded, and it predominantly acts at an azimuth of $110^{\circ}\left(290^{\circ}\right)$, the secondary directions acting at azimuths $10^{\circ}\left(190^{\circ}\right)$ and $140^{\circ}$ $\left(330^{\circ}\right)$. However, the ratios of the values of the main and secondary directions differ significantly from each other from 3:1, obtained in GrafNet/GrafNav, to 1:1.33, obtained in the Motion Tracker module. At the same time, according to the results of an analysis of the internal estimation of errors, GrafNet/GrafNav demonstrates a higher accuracy of office processing.

It has been established that short-period cyclic geodynamic movements, although they have relatively low amplitudes (up to $10-15 \mathrm{~mm}$ ), lead to the formation of fatigue effects in structures and materials, "loosening" of the rock mass that composes the adjacent massif, changes in the strength properties of soils located in the foundation of engineering structures, the manifestation of the effect of thixotropy, etc. On the other hand, in the form of short-period cyclical geodynamic movements, the unloading of the rock mass occurs, while the level of the stress-strain state, in particular its trend component, decreases, preventing its manifestation in the form of dynamic events.

An assessment of the degree of risk of destructive deformation processes was carried out on the basis of negative factors identified by geodetic and geophysical methods [7, 8]. Accordingly, a greater number of negative factors inherent in the investigated area suggests a greater probability of destructive deformation processes. The highlighted areas are divided into categories depending on the combination of negative factors influencing them.

The first category includes areas where the influence of at least three factors is manifested, among which the proximity of multidirectional structural disturbances, and increased and decreased stress concentrations are of decisive importance. Their combination indicates a higher probability of emergency events.

The second category includes areas with two operating factors, including a negative combination of structural elements of the rock mass and watering. These two factors pose a certain danger, but less in comparison with the first category. 


\section{Conclusion}

Thus, on the basis of the data obtained, the linear parameters of deformation of the rock mass and the adjacent rock mass in three planes, as well as the relative deformations and the values of the tectonic stresses that caused them, were identified. Based on the analysis of negative factors, potentially hazardous areas of the adjacent rock mass were identified.

To ensure the safe development of the Kiembaevsk field, under the conditions of established deformation processes it is required to equip observation stations and establish mine surveying control of the development of deformation processes outside the local section of the western side collapse and in potentially dangerous areas located on the limiting contour.

At the same time, in addition to what is required by the regulations, the profile lines located normal to the sides of the quarry must be provided for in the design of the observation station and the observation technique with the following:

- the ability to obtain full strain tensors; this will require the organization of an areal observation station;

- the ability to obtain data on the development of the displacement process in threedimensional space using remote systems: laser scanning, ground and air (copter) photogrammetry;

- the ability to obtain data on the hierarchically block structure of the rock mass and its changes in time - by mine surveying, geodetic and geophysical methods, carried out in the monitoring mode.

It is also necessary to regularly redefine the coordinates of the RMSN points of the open pit and dumps, linking them to the equilibrium system of the SGN, with a subsequent analysis of the development of the deformation situation and the geomechanical model of the adjacent rock mass $[9,10]$.

The paper was prepared within the framework of state assignment No. 075-00581-19-00 on topic No. 0405-2019-0007.

\section{References}

1. A.A. Panzhin, A.D. Sashurin, N.A. Panzhina, Mining information and analytical bulletin (scientific and technical journal), S24, 59-67 (2017) DOI: 10.25018/0236-1493-2017-1124-59-67.

2. A.A. Panzhin, Mining Journal, 1, 39-43 (2012)

3. B.T. Mazurov, A.A. Panzhin, A.A. Silaeva, Geodesy and Cartography, 3, 35-40 (2016) DOI: 10.22389/0016-7126-2016-909-3-25-40

4. A.A. Panzhin, Mining information and analytical bulletin, 9, 312-331 (2010)

5. A.A. Panzhin, Mine survey and subsoil use, 2(8), 43-54 (2003)

6. A.D. Sashurin, A.A. Panzhin, Ferrous metallurgy, 1(1405), 21-25 (2017)

7. A.D. Sashurin, A.A. Panzhin, A.E. Balek, S.V. Usanov, Mining Journal, 12, 16-20

(2017) DOI: 10.17580/gzh.2017.12.03

8. Yu.O. Kuzmin, Physics of the Earth, 5, 87-101 (2016) DOI:

10.7868/S0002333716050070

9. S.V. Usanov, V.V. Melnik, A.L. Zamyatin, Physical and technical problems of the development of useful minerals, 6, 83-89 (2013)

10. E.Yu. Efremov, A.A. Panzhin, T.F. Kharisov, O.D. Kharisova, Bulletin of higher educational institutions. Mining Journal, 4, 35-45 (2018) DOI: 10.21440/0536-1028-20184-35-45 